\title{
Eficácia do anestésico tópico em odontologia: Revisão de literatura
}

\section{Effectiveness of anesthetic topical in dentistry: literature review}

\begin{abstract}
RESUMO
A dor em Odontologia é uma sensação bastante desagradável, estando associada a um dano tecidual que deve ser evitado em qualquer tipo de procedimento. Estudos relacionam a dor provocada pela punção da agulha, durante a aplicação do anestésico local, com a ansiedade apresentada pelo paciente na realização do tratamento odontológico. Com isso, os anestésicos tópicos foram desenvolvidos a fim de se amenizar essa experiência dolorosa. Porém atualmente, não há uma formulação tópica capaz de eliminar completamente a dor provocada pela punção da agulha, instigando diversas pesquisas sobre eles. Este trabalho objetiva apresentar por meio de uma revisão de literatura, considerações sobre os anestésicos tópicos usados em Odontologia, abordando os aspectos relacionados à farmacologia, suas principais reações adversas, indicações e contraindicações.

Palavras-chave: Odontologia; Anestesia; Ansiedade ao Tratamento Odontológico; Odontalgia.
\end{abstract}

\begin{abstract}
Pain in dentistry is a rather unpleasant sensation, being associated with tissue damage that should be avoided in any procedure. Studies relate the pain caused by needle puncture, during the application of the local anesthetic, with the anxiety presented by the patient in performing the dental treatment. Thus, topical anesthetics were developed to alleviate this painful experience. Currently, there is no topical formulation of drug capable of eliminating the pain caused by needle puncture, instigating various research on them. This work aims to present, through a review of the literature, considerations about topical anesthetics used in dentistry, addressing aspects related to pharmacology, the main adverse reactions, indications and contraindications.
\end{abstract}

Key-words: Odontology; Anesthesia; Anxiety to Dental Treatment; Toothache.

\section{NTRODUÇÃO}

A dor em Odontologia é uma sensação bastante desagradável e que deve ser evitada em qualquer tipo de procedimento odontológico realizado. ${ }^{1}$ Os anestésicos tópicos foram desenvolvidos durante a segunda metade do século XIX, inicialmente como o uso tópico de cocaína. No entanto, foi necessário quase um século inteiro para uma efetiva e segura utilização dos anestésicos tópicos. ${ }^{2}$

Umas das principais propriedades para um bom anestésico são: baixa toxicidade, não ser irritante para os tecidos e não lesionar as estruturas nervosas. Além disso, sua ação deve ter início rápido, duração eficaz para o procedimento
Fernanda Britto de Melo Silva ${ }^{1}$

Fernanda Ramalho Tuorto ${ }^{2}$

Isabelly Pereira Vasconcellos ${ }^{3}$

Olivio Cesar de Melo Silva ${ }^{4}$

Rodrigo Figueiredo de Brito Resende

${ }^{1}$ Graduada em Odontologia pela Universidade Federal Fluminense, Niterói / RJ, Brasil e Residente do Serviço de Cirurgia e Traumatologia Buco-Maxilo-Facial da Universidade Federal Fluminense, Niterói / RJ, Brasil.

${ }^{2}$ Graduanda em Odontologia pela Universidade Federal Fluminense, Niterói / RJ, Brasil.

3 Graduada em Odontologia pela Universidade Federal Fluminense, Niterói / RJ, Brasil e aluna do curso de especialização em Implantodontia da Universidade Federal Fluminense, Niterói / RJ, Brasil.

${ }^{4}$ Especialista em Cirurgia e Traumatologia Buco-Maxilo-Facial pela Universidade Federal do Rio de Janeiro/ RJ, Brasil e Especialista em Implantodontia pela Universidade Estadual do Rio de Janeiro/ RJ, Brasil.

${ }^{5}$ Doutor em Odontologia pela Universidade Federal Fluminense, Niterói / RJ, Brasil e professor da disciplina de Cirurgia oral menor e Anestesiologia da Faculdade de Odontologia da Universidade Federal Fluminense, Niterói / RJ, Brasil.

Endereço para correspondência:

Fernanda Britto de Melo Silva

Rua Ministro Viveiros de Castro, 82/ 601 Copacabana - Rio de Janeiro - RJ - 22021-010, Brasil.

E-mail: fernandabrittodemelo2@hotmail.com Telefone: +5521998177665 
e ser reversível. A amida e o éster são os tipos de anestésicos mais utilizados na Odontologia, sendo a primeira menos tóxica, mais eficaz e com menor potencial alergênico do que os ésteres. ${ }^{3}$

Segundo a última publicação da Organização Mundial de Saúde (OMS) em 2012 para o tratamento da dor, este envolve a utilização de analgésicos opioides e não opióides, anti-inflamatórios não esteroidais (AINE's) e medicações adjuvantes, a depender do tipo em questão: leve, moderada e intensa. Estes fármacos, tradicionalmente, são administrados por via sistêmica ou no neuroeixo, porém, quando aplicados por estas vias, estão associados a efeitos colaterais importantes, podendo assim inviabilizar o seu uso. ${ }^{4}$

Por definição, formulações tópicas são aquelas que, quando aplicadas na proximidade da área afetada, exercem ação analgésica estando associadas ao aumento da concentração no tecido alvo e à reduzida concentração sérica. Dessa forma, os fármacos de administração tópica exercem seus efeitos na periferia, próximo ao seu sítio de aplicação, reduzindo as chances da ocorrência de efeitos colaterais indesejáveis. ${ }^{4}$

Os anestésicos locais podem desencadear reações alérgicas dos tipos I (hipersensibilidade imediata) e IV (dermatite de contato), sendo que na utilização tópica de anestésicos locais, o segundo tipo é observado mais frequentemente que as reações alérgicas mediadas por anticorpos. ${ }^{5}$

Outra complicação que pode ser vista com o uso tópico dos anestésicos locais, é a metahemoglobinemia. Onde a prilocaína e a benzocaína, possuem diversos relatos descritos, devendo ser evitados em pacientes portadores de insuficiência cardíaca, respiratória ou doenças metabólicas e em gestantes, por causa do risco do feto vir a contrair a doença. ${ }^{6,7}$

A eficácia dos anestésicos tópicos tem sido muito aprimorada com a tecnologia de lipossomas. Os lipossomas foram descobertos por Bangham em 1963 e consistem de moléculas esféricas, formadas pela interação de lipídios suspensos numa fase aquosa que, devido à diferença de polaridade com o meio, tendem a se agrupar, formando vesículas. ${ }^{8,9}$ Essas moléculas são biocompatíveis, biodegradáveis, possuem baixo risco de toxicidade, imunogenicidade e antigenicidade. ${ }^{9}$ Tal tecnologia tem como mecanismo a liberação mais lenta da droga, prolongando a duração da anestesia e reduzindo a toxicidade para o sistema nervoso central e cardiovascular. ${ }^{10}$

\section{REVISÃO DE LITERATURA}

A dor em Odontologia é uma situação bastante desagradável e está ligada diretamente a dois fenômenos: aos aspectos psicológicos/emocionais em relação a dor e a sensação de dor propriamente dita. A sensação dolorosa impede ao profissional executar, em detalhes, o seu trabalho provocando temor e desconforto ao paciente. ${ }^{1}$

A ansiedade ao tratamento dentário pode estar relacionada a diversos fatores, tais como medo da dor provocada pelo procedimento ao qual o paciente será submetido, medo de procedimentos que envolvam sangue, ou pode ser devido a experiências traumáticas prévias no consultório odontológico, principalmente na infância. ${ }^{11}$ Pacientes ansiosos tendem a experimentar a dor em um período maior de tempo, nos procedimentos odontológicos, quando comparado aqueles sem ansiedade. Dessa forma, Wijk \& Hoogstraten ${ }^{12}$ 
(2009) estabeleceram uma relação entre a ansiedade durante o tratamento odontológico e uma saúde oral deficiente.

Estudos relacionam diretamente a ansiedade no tratamento odontológico com a dor provocada pela punção da agulha durante a aplicação do anestésico local, independente do procedimento realizado ou da faixa etária avaliada. ${ }^{2,12}$ Assim, a utilização dos métodos capazes de diminuir a dor provocada pela punção da agulha durante a injeção anestésica ou de outros procedimentos invasivos na mucosa bucal, resultaria em uma diminuição da ansiedade no paciente e seriam observados melhores índices de saúde oral. ${ }^{2}$ Nesse sentido, os anestésicos locais de uso tópico atuam como preparação da mucosa para aliviar o efeito introdutor da agulha em infiltrações anestésicas. ${ }^{1}$

O conhecimento sobre anestésicos locais é muito importante uma vez que o uso destas substâncias, sem um critério científico consistente, pode levar o paciente ao óbito por superdosagem, sensibilidade aguda e depressão do sistema nervoso central. ${ }^{13}$ Carvalho B et al. ${ }^{3}$ (2013) consideram como propriedades essenciais para um bom anestésico: baixa toxicidade, não ser irritante aos tecidos, não lesionar as estruturas nervosas, sua ação deve ter início rápido, duração eficaz para o procedimento e ser reversível.

Farmacologicamente, sabe-se que os analgésicos têm por mecanismo de ação principal a atuação em sítios específicos localizados no sistema nervoso central e na periferia. Tal constatação impulsionou a realização de trabalhos que propunham a administração tópica de anti-inflamatórios não esteroidais, anestésicos locais, capsaicina, antidepressivos tricíclicos, cetamina, clonidina, opioides e canabinoides. ${ }^{4}$ Esses estudos mostraram que a aplicação tópica destes fármacos possibilita elevadas concentrações nos sítios efetores periféricos em contraposição a baixos níveis séricos dos mesmos, reduzindo o risco da ocorrência de efeitos colaterais indesejáveis. ${ }^{4}$

O mecanismo de ação está baseado na diferença da concentração de sódio e potássio nos fluidos intra e extracelulares, que estabelece gradiente iônico entre os meios, mantido pela bomba $\mathrm{Na}^{+} / \mathrm{K}^{+}$ATPase. ${ }^{14}$ Em repouso, o meio extracelular é positivo em relação ao intracelular. Quando há estímulo, a membrana aumenta sua permeabilidade ao sódio, causando despolarização temporária. O repouso é restabelecido pela diminuição da permeabilidade ao sódio e pela atividade da $\mathrm{Na}^{+} / \mathrm{K}^{+}$ATPase, que restabelece o gradiente inicial. Os anestésicos tópicos inibem a despolarização da fibra, impedindo a transmissão do impulso e determinando perda das sensações, mas sem alterar o nível de consciência. ${ }^{14}$

Como podemos observar no estudo de Froes GC et al. ${ }^{14}$ (2010), os anestésicos tópicos, bioquimicamente, se dividem em dois grupos: ésteres e amidas. Sua estrutura básica consiste num anel aromático lipofílico e em um grupo amina hidrofílico conectado por uma cadeia intermediária. Esta cadeia é o que classifica os anestésicos em ésteres ou amidas. A diferença mais expressiva entre as duas classes é a estabilidade química. Os ésteres são relativamente instáveis em solução e facilmente metabolizados no plasma pela pseudocolinesterase e um dos seus principais metabólitos é o ácido paraminobenzóico (PABA), o qual tem considerável potencial alergênico. As amidas são mais estáveis, têm metabolização no fígado e menor capacidade de sensibilização. ${ }^{14}$

Quando o anestésico é aplicado topicamente na mucosa oral, geralmente produz anestesia nos 2 ou 3 milímetros mais superficiais desse tecido e por essa razão é utilizado na odontologia para reduzir a dor provocada pela inserção da agulha ou em outros pro- 
cedimentos pouco invasivos. A eficácia desse tipo de anestésico é determinada pelo tempo de aplicação na mucosa, sendo recomendada a aplicação por no mínimo dois minutos. ${ }^{15}$

Ogle OE \& Mahjoubi $G^{15}$ (2012) dizem que a concentração de anestésico tópico é tipicamente maior do que quando utilizado na forma injetável, uma vez que são incapazes de atravessar a mucosa intacta. Dessa forma, quanto maior a concentração, maior a difusão do anestésico no tecido. Entre os principais anestésicos tópicos estão: a Benzocaína, a Lidocaína e, mais recentemente, o creme EMLA ${ }^{\circledR}$. A Benzocaína é um dos anestésicos tópicos mais utilizados na Odontologia e por ser pouco hidrossolúvel permanece no tecido onde é aplicada. ${ }^{15}$

Conforme Malamed SF${ }^{16}$ (2013), alguns cuidados, no entanto, devem ser tomados na utilização da benzocaína, uma vez que é um éster e pode produzir reações alérgicas em algumas pessoas. Seu uso está contraindicado em crianças com idade inferior a dois anos pelo risco de provocar metemoglobinemia, uma síndrome que resulta em hipóxia tecidual e em casos mais graves pode levar ao óbito. Prilocaína, articaína e benzocaína (uso tópico) são considerados os anestésicos que mais causam metemoglobinemia, devendo ser evitados em grandes cirurgias, portadores de insuficiência cardíaca, respiratória ou doenças metabólicas e em gestantes, por causa do risco do feto vir a contrair a doença. ${ }^{6}$

A lidocaína também é utilizada como anestésico tópico, em concentrações que geralmente variam entre 2 a 10\% ou em combinação com outros anestésicos locais. ${ }^{2}$ Conforme trabalho de Froes GC et al. ${ }^{14}$ (2010) a lidocaína, assim como os demais anestésicos do tipo amida, pode causar reação alérgica de tipo grave. A absorção sistêmica da lidocaína causa efeitos adversos dose-dependentes. Os primeiros sintomas da toxicidade incluem: zumbido, parestesia perioral, gosto metálico, sonolência, posteriormente agitação e apreensão, devido ao comprometimento do sistema nervoso central. Em seguida pode ocorrer convulsão tônico-clônica generalizada, e, em estágio mais avançado, depressão cardiovascular e respiratória, além de arritmias. ${ }^{14}$

O creme EMLA ${ }^{\circledR}$ (Eutetic Mixture of Local Anesthetics) é uma mistura de lidocaína $(2,5 \%)$ e prilocaína $(2,5 \%)$ e foi desenvolvido com o intuito de diminuir ou eliminar a dor durante procedimentos realizados superficialmente na pele intacta. ${ }^{14}$ No entanto, esta formulação apresenta algumas desvantagens para uso em mucosa bucal, como gosto desagradável, baixa viscosidade e dificuldade de retenção no sítio de aplicação por ser uma formulação desenvolvida para uso em pele. ${ }^{17}$ Além disso, pode provocar efeitos adversos sobretudo locais e pode causar também prurido, queimação e púrpura. $\mathrm{O}$ pH alcalino do creme pode lesionar a mucosa conjuntival e a dermatite de contato alérgica, quando ocorre, deve-se em geral à prilocaína. ${ }^{14}$ Essa substância ainda pode causar metahemoglobinemia e toxicidade do sistema nervoso central. Estes eventos foram observados em crianças que receberam EMLA ${ }^{\circledR}$ para curetagem de lesões cutâneas. Dessa forma, atualmente esse creme encontra-se com a fabricação temporariamente descontinuada no Brasil, sendo notificada a ANVISA em dezembro de 2016. ${ }^{18}$

Dentre os procedimentos em que se tem testado o uso dos anestésicos tópicos estão a raspagem e alisamento radicular. Dessa forma, autores têm proposto o uso de substâncias anestésicas de aplicação tópica para anestesia durante raspagem e alisamento radicular. ${ }^{19}$

Um dos efeitos adversos provocado pelos anestésicos mais conhecido é a reação anafilática. "A reação anafilática a um medicamento é qualquer reação mediada pelo anti- 
corpo IgE formado especificamente contra essa droga ou um de seus metabólitos". ${ }^{5}$ Araújo LMT \& Amaral JLG ${ }^{5}$ (2004) afirmam que os anestésicos locais podem desencadear reações alérgicas dos tipos I (hipersensibilidade imediata) e IV (dermatite de contato). Já, com o uso tópico de anestésicos locais, a reação mais frequentemente observada é a dermatite de contato, sendo mais raras as reações alérgicas mediadas por anticorpos.

Um recurso que vem sendo estudado a fim de se aprimorar a eficácia dos anestésicos tópicos é a tecnologia de lipossomas. Os lipossomas encapsulados com Lidocaína são efetivos na redução da dor à inserção da agulha na pele. ${ }^{20} \mathrm{~A}$ anestesia tópica lipossomal também tem sido testada na mucosa oral e provou ser eficaz na anestesia da mucosa bucal antes da injeção, por exemplo: Ropivacaína 1\% e 2\% encapsulada em lipossomos. Tendo em vista a melhoria na segurança do procedimento anestésico, a associação de anestésicos locais e novos sistemas de liberação controlada tem sido alvo de estudos. ${ }^{20}$

Descobertos por Bangham em 1963, os lipossomos são biocompatíveis, biodegradáveis, com risco reduzido de toxicidade sistêmica ou local, imunogenicidade e antigenicidade, principalmente pela semelhança de seus monômeros constituintes (fosfatildilcolina e colesterol) com os das membranas biológicas. ${ }^{9} \mathrm{O}$ uso clínico do sistema lipossomal para anestesia local em medicina vem confirmando as vantagens terapêuticas desta associação. Em Odontologia, o uso de preparações lipossomais possibilita a manutenção da duração e da efetividade da anestesia, dispensando-se o uso dos vasoconstritores. ${ }^{7}$

Além da questão da dor associada à punção da agulha na anestesia local e da possibilidade de uma reação anafilática existem outros riscos. Entre os riscos associados com as injeções, o da infecção, provavelmente, é o que demanda mais cuidado. Fortunato RX ${ }^{21}$ (1990) diz que embora se precavendo com a utilização de solução e agulha estéreis, pode-se questionar o risco de se transportar microrganismos com a agulha para o interior dos tecidos. Brancher T et al. ${ }^{22}$ (2010) realizaram um trabalho cujo objetivo foi avaliar a atividade antimicrobiana de diferentes marcas comerciais de anestésicos tópicos frente a diferentes microrganismos encontrados na cavidade bucal em uma situação de saúde, e também em relação a um microrganismo exógeno. Os resultados obtidos nesse trabalho possibilitaram concluir que alguns anestésicos tópicos utilizados (Benzotop ${ }^{\circledR}$ nos três sabores disponíveis - pinacolada, tutti-frutti e menta- e Lidocaína 5\%) com a função prioritária de redução da dor podem ser utilizados também com função de inibição do crescimento bacteriano. Porém, nenhum dos anestésicos tópicos testados foi capaz de inibir o crescimento do fungo C. albicans. ${ }^{22}$

Segundo Brancher T et al. ${ }^{22}$ (2010) o uso dos anestésicos tópicos aumentou significativamente nos últimos anos, em procedimentos médicos e, de forma indiscriminada, pela população. Isso causa preocupação quanto aos possíveis riscos da utilização indevida.

A seleção do anestésico tópico apropriado irá depender, então, das características do fármaco, do veículo utilizado e do tipo de superfície em que seja aplicado. ${ }^{14}$

\section{MATERIAL E MÉTODOS}

Foi realizada uma revisão de literatura, com o objetivo de apresentar considerações sobre os anestésicos tópicos utilizados em Odontologia, abordando aspectos relativos à farmacologia, às principais reações adversas, às indicações e contraindicações. Em relação 
ao levantamento bibliográfico, foram realizadas consultas nas bases de dados PubMed, Scielo BVS e Google Acadêmico, com os seguintes critérios de inclusão: dissertações, artigos e livros publicadas no idioma português, inglês e espanhol que tenham como ênfase a eficiência, a farmacologia, o mecanismo de ação, indicação e contraindicações dos anestésicos tópicos, além de testes com diferentes tipos dos mesmos. Os critérios de exclusão utilizados no estudo foram: artigos em outras línguas que não o português, espanhol ou inglês. Os termos utilizados para a busca foram: anestesia tópica (topical anesthesia), anestésicos locais (local anesthetics), ansiedade em odontologia (anxiety in dentistry) e dor em odontologia (Pain in dentistry).

Foram encontrados 43 artigos e, considerando os critérios de inclusão, foram incluídos para análise 25 artigos, 3 dissertações, 1 tese e 1 livro. Os artigos, as dissertações, a tese e o livro usados para análise têm data de publicação compreendida no período entre o ano de 1990 e 2019.

\section{DISCUSS ÃO}

Pode-se perceber que a ansiedade está muito relacionada ao tratamento odontológico, sobretudo quando se trata da realização de procedimentos que envolvam anestesia local. O medo da dor é a maior causa de apreensão ao tratamento. Assim, a anestesia local, modo principal de controle da dor transoperatória, por si só pode provocar grande ansiedade. ${ }^{23}$

Freitas VS² (2014) corrobora com Zanatta FB et al. ${ }^{24}$ (2010), uma vez que estabelecem uma ligação entre a ansiedade ao tratamento odontológico com uma saúde oral pobre, o que pode representar um problema de saúde pública. Para esses autores, em procedimentos de raspagem e alisamento radicular é desejável uma modalidade anestésica de ação rápida, de fácil aplicação e indolor. ${ }^{2}{ }^{17}$ A mais comumente utilizada pelos clínicos é a anestesia injetável (troncular/infiltrativa) combinada ou não com anestesia tópica. ${ }^{19}$ Porém, a anestesia injetável pode induzir a ansiedade e medo além de ser um motivo para o paciente evitar o tratamento odontológico. ${ }^{25}$ Milgrom P et al. ${ }^{26}$ (1997) complementam, concluindo que $5 \%$ dos pacientes indicados para tratamento periodontal evitaram, cancelaram ou não compareceram a consulta odontológica por receio a anestesia injetável.

Também para Amorim KS7 (2016) a anestesia local, mesmo sendo a forma mais eficaz no controle da dor em odontologia, por si só já é associada a ansiedade e medo.

Dessa forma, Freitas VS² (2014) afirma que uma diminuição da ansiedade no paciente seria possível a partir do uso de métodos capazes de diminuir a dor provocada pela punção da agulha durante a injeção anestésica ou de outros procedimentos invasivos na mucosa bucal e, consequentemente, resultaria em melhores índices de saúde oral.

A ansiedade e o medo de sentir dor provocados pelos procedimentos odontológicos que envolvem anestesia local podem ser amenizados com o uso de anestésicos tópicos. Essa alternativa anestésica que precede a anestesia local pode ser, muitas vezes, eficaz na redução da dor da introdução da agulha e da injeção da solução anestésica. ${ }^{2} \mathrm{O}$ estudo feito por Rosivack $\mathrm{RG}^{27}$ (1990) mostrou que a anestesia tópica diminuiu a dor e o desconforto causado pela puntura da agulha e por outros procedimentos dentais seletos potenciais.

Todos os artigos analisados que falam sobre anestesia tópica convergem entre si ao se referirem à região de mucosa palatina como a região mais dolorosa no indivíduo ao 
receber uma infiltração local. Pode-se dizer, assim, que a dor durante a punção é profundamente influenciada pela área anestesiada, uma vez que injeções palatais e na região anterior da maxila são muito desconfortáveis. ${ }^{25}$ Autores explicam que esse fato está relacionado à rigidez e íntima ligação da mucosa do palato com o periósteo subjacente e seu abundante suprimento nervoso dizendo que a grande aderência da mucosa do palato, contribui de forma decisiva para uma maior sensibilidade nas infiltrações anestésicas nessa região. ${ }^{1,7}$

A área fibrosa ou periférica do palato é difícil e dolorida devido à necessidade de exercer pressão para conseguir a penetração do líquido anestésico na profundidade dos tecidos. ${ }^{1}$ Tal afirmação corrobora com Amorim KS ${ }^{7}$ (2016), que diz que a melhora nos agentes e as inovações técnicas para anestesia local são, provavelmente, o mais importante avanço acontecido nos últimos anos na odontologia. São utilizados, então, alguns artifícios, como: administração sob pressão; resfriamento do palato; baixa velocidade de injeção no palato e também o uso de sistemas de injeção computadorizados.

Um grande número de técnicas tem sido usado com o propósito de gerar o alívio da dor para as injeções palatais por técnica anestésica odontológica, dentre elas está a anestesia tópica. Entretanto, estas técnicas não conseguem suprimir por completo a dor no momento da injeção palatal. ${ }^{7}$

Franz-Montan M et al. ${ }^{28}$ (2012) avaliaram a eficácia de formulações lipossomais de Ropivacaina 1 e $2 \%$ no alívio da inserção da agulha e da injeção anestésica no palato de 40 voluntários. Os anestésicos tópicos permaneceram por 5 minutos na mucosa antes de serem removidos e foi então injetada uma solução de $2 \%$ de lidocaína contendo epinefrina. As formulações com ropivacaína não reduziram a dor da punção e nem a da injeção anestésica no palato quando comparados com o grupo placebo. Já o EMLA ${ }^{\circledR}$, utilizado como controle positivo no estudo, somente foi eficaz em reduzir a dor da inserção da agulha, sem qualquer efeito significante na redução da dor provocada pela injeção da solução do anestésico. ${ }^{28}$

Diversos fatores podem estar relacionados a esses resultados conflitantes referentes à eficácia dos anestésicos tópicos em amenizar a dor da punção e da injeção anestésica, dentre eles estão: a diferença entre o local de aplicação, a secagem prévia da região, o tipo de anestésico utilizado, o tempo de aplicação, a agulha utilizada e o método utilizado para avaliar a dor. ${ }^{2}$

Amorim $\mathrm{KS}^{7}$ (2016) e Freitas $\mathrm{VS}^{2}$ (2014) convergem quanto ao anestésico tópico $\mathrm{EMLA}^{\circledR}$. Esse último é uma formulação mais recente, uma mistura eutética de lidocaína a $2,5 \%$ e prilocaína a $2.5 \%$, que mostrou resultados mais promissores logo nos primeiros estudos realizados aplicando o agente na mucosa palatina. Apesar de não possuir indicação como anestésico tópico oral, o EMLA ${ }^{\circledR}$ foi testado inicialmente na odontologia com a intenção de reduzir a dor provocada pela punção da injeção durante a anestesia por Holst \& Evers em 1985..$^{2}$ Porém, Freitas VS² (2014) quanto Flores MP et al. ${ }^{4}$ (2012) destacam que não se deve descartar a possibilidade de ocorrer metemoglobinemia e ulcerações na mucosa gengival, devido à prilocaína, assim como Gobbato CARS ${ }^{18}$ (2019) inclui como efeito adverso desse anestésico a metahemoglobinemia e a toxicidade do sistema nervoso central. Essa substância, então, teve sua fabricação temporariamente descontinuada no Brasil.

Olopes GLC ${ }^{29}$ (2018) comparou a Benzocaína com outro anestésico tópico: o Cloridrato de Tetracaína. Constatou que ambos foram eficazes na substituição da anestesia infiltrativa para a instalação do grampo de isolamento absoluto, para a realização de se- 
lante resinoso em fóssulas e fissuras. Porém a Benzocaína tem um tempo de duração da ação de até quinze minutos. Já a Tetracaína é o mais potente entre os anestésicos tópicos com duração de ação de até sessenta minutos. Isso devido à sua estrutura cilíndrica e por ser hidrofóbica, que a permite penetrar mais profundamente que outros anestésicos, uma vez que consegue desorganizar a bicamada lipídica dos tecidos.

Portanto, inserir o Cloridrato de Tetracaína na rotina da clínica odontológica é uma alternativa segura e viável no controle da dor, proporcionando maior conforto e consequentemente controlando o medo e ansiedade dos pacientes. Além disso, evitar-se a aplicação de anestesia infiltrativa é um ganho em tempo de trabalho, segurança e conforto do paciente. ${ }^{29}$

Nos últimos tempos, observamos muitos questionamentos a respeito do aprimoramento dos anestésicos tópicos com a tecnologia dos lipossomas. Um estudo feito por Friedman PM et al. ${ }^{30}$ (2001) comparou a profundidade e duração da anestesia produzida por quatro anestésicos tópicos: EMLA $^{\circledR}$, ELAMax (Lidocaína 4\% em veiculo lipossomal), Betacaína-LA (Lidocaína + Prilocaína + vasoconstritor) e Tetracaína 4\% em gel. Os testes foram realizados em 10 locais do antebraço de 12 voluntários, através de estimulação com laser. Os resultados mostraram superioridade da Lidocaína lipossomal na promoção da anestesia em relação às outras preparações.

Outro exemplo de estudo mostrado por Amorim $\mathrm{KS}^{7}$ (2016) foi em técnica infiltrativa na maxila na qual houve aumento da duração de ação do anestésico local encapsulado em lipossomas.

Apesar de todos os testes, pesquisas e estudos, é importante enfatizar que, até o momento, não há anestésico tópico capaz de eliminar totalmente o desconforto provocado pela agulha durante a injeção anestésica. Dessa forma, os profissionais da saúde, em particular, Cirurgiões-Dentistas, devem estudar e se atualizar sempre, pois o ser humano é único e complexo e devemos zelar por sua integridade física e mental. ${ }^{29}$

\section{CONCLUSÃO}

Por meio desta revisão de literatura podemos concluir que o conhecimento sobre os sais anestésicos, a técnica correta empregada, a condição sistêmica do paciente e o controle de ansiedade prévia ao procedimento são necessários para um atendimento odontológico seguro e de qualidade. Além disso, foi possível concluir que nenhum anestésico tópico desenvolvido até o presente momento é capaz de eliminar, comprovada e totalmente, a dor nem o incômodo da punção da agulha em procedimentos odontológicos.

Dessa forma, objetivando-se uma odontologia com o mínimo de desconforto, indolor e rápida, os cirurgiões-dentistas necessitam aprimorar-se cada vez mais nas técnicas anestésicas e cumprir todas as etapas para uma analgesia local segura e eficiente.

\section{REFERÊNCIAS BIBLIOGRÁFICAS}

1. Vasconcelos BCE, Lago CAP, Lago LM, Silva MBL. Avaliação da sintomatologia dolorosa nas anestesias infiltrativas no palato quando utilizado a xilocaína a 5\% e/ou placebo (vaselina) como anestésico tópico: estudo piloto. Rev. Odonto. Ciênc. - PUCRS. 2006;21(51):82-86. Disponível em: http://bases.bireme.br/cgi-bin/wxislind.exe/iah/online/?IsisScript=iah/iah.xis\&src=google\&base=AD OLEC\&lang=p\&nextAction=lnk\&exprSearch=495247\&indexSearch=ID 
2. Freitas VS. Desenvolvimento e avaliação da eficiência de um bioadesivo contendo extrato de spilanthes acmella 1. murray para administração oral como anestésico tópico [dissertação em CD-ROM]. Piracicaba: Universidade Estadual de Campinas; 2014. Disponível em: http://repositorio. unicamp.br/jspui/handle/REPOSIP/288757

3. Carvalho B, Fritzen EL, Parodes AG, Santos RB, Gedoz L. O emprego dos anestésicos locais em odontologia: revisão de literatura. Rev. bras. odontol. 2013;70(2):178-81. Disponível em: http:// revodonto.bvsalud.org/scielo.php?pid=S0034-72722013000200016\&script=sci_arttext].

4. Flores MP, de Castro APCR, Nascimento JS. Analgésicos tópicos. Reve. Bras. Anestesiol. 2012;62(2):244-252. Disponível em: https://repositorio.unesp.br/handle/11449/73204].

5. Araújo LMT, Amaral JLG. Alergia à lidocaína. Relato de caso. Rev. Bras. Anestesiol. 2004;54(5):672-676. Disponível em: http://www.scielo.br/pdf/rba/v54n5/v54n5a08.pdf

6. Paiva LCA, Cavalcanti AL. Anestésicos Locais em odontologia: uma revisão de literatura. Publ. UEPG Ci. Biol. Saúde.2005;11(2):35-42. Disponível em: http://www.revistas2.uepg.br/index.php/ biologica/article/viewFile/414/417].

7. Amorim KS. Comparação da eficácia anestésica entre dois géis em exodontias de molares superiores: Ensaio clínico randomizado [dissertação em CD-ROM]. Aracaju: Universidade Federal de Sergipe; 2016. Disponível em: http://ri.ufs.br/jspui/handle/riufs/5879

8. Banerjee R. Liposomes: applications in medicine. J Biomat App. 2001;16(1):3-21. Disponível em: https://journals.sagepub.com/doi/10.1106/RA7U-1V9C-RV7C-8QXL

9. Grant GJ et al. A novel liposomal bupivacaine formulation to produce ultralong-acting analgesia. Anesthesiology. 2004;101(1):133-7. Disponível em: https://www.ncbi.nlm.nih.gov/pubmed/15220782]. 10. Araujo DR, Pinto LMA, Braga AFA, de Paula E. Formulações de anestésicos locais de liberação controlada: aplicações terapêuticas. Rev. Bras. Anestesiol. 2003;53(5):653-6. Disponível em: http:// www.scielo.br/scielo.php?pid=S0034-70942003000500014\&script=sci_abstract\&tlng=pt

11. Hmud R, Walsh LJ. Dental anxiety: causes, complications and management approaches. J Minim Interv Dent. 2009;9(5):67-78. Disponível em: https://pdfs.semanticscholar.org/7269/d14d5cb034fefe632a609eb0dc354f226341.pdf

12. Wijk AJ, Hoogstraten J. Anxiet and pain during dental injections. J Dent. 2009;37(9):700-4. Disponível em: https://www.ncbi.nlm.nih.gov/pubmed/19556053

13. Montan MF, COGO K, Bergamaschi CC, Volpato MC, Andrade ED. Mortalidade relacionada ao uso de anestésicos locais em odontologia. RGO. 2007;55(2):197-202. Disponível em: https:// s3.amazonaws.com/academia.edu.documents/46938766/RGO-2007-570.pdf?AWSAccessKeyId=A KIAIWOWYYGZ2Y53UL3A\&Expires=1559097664\&Signature=\%2BNwLHLYhOiL5Y\%2BFfSgU9A aBq\%2FYM\%3D\&response-content-disposition=inline \%3B\%20filename\%3DMortalidade_relacionada_ao_uso_de_aneste.pdf

14. Froes GC, Ottoni FA, Gontijo G. Anestésicos tópicos. Surg. Cosmet. Dermatol. 2010;2(2):111-16. Disponível em: https://www.redalyc.org/html/2655/265521080007/

15. Ogle OE, Mahjoubi G. Local anesthesia: agents, techniques and complications. Dent. Clin. North. Am. 2012;56(1):133-148. Disponível em: https://www.ncbi.nlm.nih.gov/pubmed/22117947

16. Malamed SF. Manual de anestesia local. 6aed. São Paulo: Elsevier; 2013: p.3-26.

17. de Sousa HMX. Filmes mucoadesivos à base de quitosana e pectina contendo anestésicos locais: avaliação da permeação e mucoadesão in vitro visando aplicação tópica em odontologia [dissertação em CD-ROM]. Piracicaba: Faculdade de Odontologia de Piracicaba da Universidade Estadual de Campinas; 2018. Disponível em: http://repositorio.unicamp.br/jspui/handle/REPOSIP/331501

18. Gobbato CARS. Farmacocinética, eficácia e segurança de uma nova formulação tópica nanotecnológica de lidocaína $25 \mathrm{mg} / \mathrm{g}$ e prilocaína $25 \mathrm{mg} / \mathrm{g} \mathrm{em}$ pacientes adultos e pediátricos [tese em CD-ROM]. Campinas: Faculdade de Ciências Médicas da Universidade Estadual de Campinas; 2019. Disponível em: http://repositorio.unicamp.br/jspui/handle/REPOSIP/334212

19. Van Steenberghe D, Bercy P, De Boever J, Adriaens P, Geers L, Hendrickx E.Patient evaluation of a novel non-injectable anesthetic gel: a multicenter crossover study comparing the gel to infiltration anesthesia during scaling and root planing (2004) apud Zanatta FB, Cargnelutti B, Freitas DN, Antoniazzi RP. Avaliação de um anestésico tópico contendo prilocaína e lidocaína para raspagem subgengival [dissertação em CD-ROM]. Santa Maria: Centro Universitário Franciscano; 2010.

20. Eichenfield et al. A clinical study to evaluate the efficacy of ELA-Max (4\% Liposomal Lidocaine) as compared with eutectic mixture of local anesthetics cream for pain reduction of venipuncture in children pediatrics. Pediatrics. 2002;109(6): 1093-9. Disponível em: https://www.ncbi.nlm.nih.gov/ pubmed/12042548 
21. Fortunato, RX. Avaliação da atividade antimicrobiana de soluções anestésicas tópicas comerciais. Estudo "in vitro" e "in vivo" [tese em CD-ROM] Piracicaba: Universidade Estadual de Campinas; 1990. Disponível em: http://repositorio.unicamp.br/jspui/handle/REPOSIP/289387 22. Brancher T, Mattis P, Silva RM, Holderbaum RM, Rockenbach MIB. Avaliação da atividade antimicrobiana de anestésicos tópicos disponíveis no comércio brasileiro [ CD-ROM ]. In: XI Salão de Iniciação Científica PUCRS; 2010 Ago 09-12; Rio Grande do Sul. Anais. Rio Grande do Sul: XI Salão de Iniciação Científica PUCRS; 2010.

23. Guerra AAS, Souza LMA, Ribeiro AO, Ramacciato JC, Motta RHL, Meechan JG. Avaliação do desconforto palatal após diferentes técnicas de infiltração vestibular de articaína na maxila. Pesqui. bras. odontopediatria clín. integr. 2012;12(1):27-31. Disponível em: https://www.redalyc.org/html/637/63723468004/

24. Zanatta FB, Cargnelutti B, Freitas DN, Antoniazzi RP. Avaliação de um anestésico tópico contendo prilocaína e lidocaína para raspagem subgengival [dissertação em CD-ROM]. Santa Maria: Centro Universitário Franciscano; 2010.

25. Vickers ER, Punnia-Moorthy A. A clinical evaluation of three topical anaesthetic agents. Aust. Dent. J. 1992;37:267-270.Disponível em: https://www.ncbi.nlm.nih.gov/pubmed/1444945

26. Milgrom P, Coldwell SE, Getz T, Weinstein P, Ramsay DS. Four dimensions of fear of dental injections. J Am Dent Assoc. 1997;128:756-766. Disponível em: https://www.ncbi.nlm.nih.gov/pubmed/9188235

27. Rosivack RG, Koenigsberg SR, Maxwell KC. An analysis of the efectiveness of two topical anesthetic. Anesth. Prog. 1990;37:290-2. Disponível em: https://www.ncbi.nlm.nih.gov/pmc/articles/PMC2162554/

28. Franz-Montan M, Paula E, Groppo FC, Silva ALR, Ranali J, Volpato MC. Liposomal delivery system for topical anaesthesia of the palatal mucosa. Br J Oral Maxillofac Surg. 2012;50:60-4. Disponível em: https://www. sciencedirect.com/science/article/abs/pii/S0266435610003517

29. Olopes GLC. Estudo Comparativo Entre Dois Anestésicos Tópicos para colocação de grampo no isolamento absoluto [dissertação em CD-ROM]. São José dos Campos: Universidade Estadual Paulista (UNESP); 2018. Disponível em: https://repositorio.unesp.br/handle/11449/157451

30. Friedman PM, Mafong EA, Friedman ES, Geronemus RG: Topical anesthetics update: EMLA and beyond. Dermatol Surg. 2001;27:1019-2001. Disponível em: https://onlinelibrary.wiley.com/doi/abs/10.1046/j.15244725.2001.01855.x 